\title{
Method of the Year 2008: cast your vote!
}

\author{
You can now nominate candidates and vote online to help select the Method of the Year 2008.
}

When Nature Methods' editors sat down last year to select a Method of the Year, it was with the firm intention of making this affair a yearly tradition. It is now time to gear up for the Method of the Year 2008, and this year, we are asking for your opinion. You can nominate candidate methods as well as vote and comment on posted suggestions on the new websitehttp://www.nature.com/nmeth/votemoy2008.

The Method of the Year event is a celebration of methods development and innovation because we think that methods developers should have their share of the limelight. It is also a fun opportunity to assemble Commentaries, technical information and news items about a method we consider particularly important among the developments that we, as editors, continuously observe across a broad range of disciplines. But we also wanted to take the pulse at the bench and see what you, with firsthand experience, think of recent methods developments. This online voting and nomination process is your opportunity to speak up. We will weigh our decision in light of the popular vote.

We are interested in methods that have come into their own in 2008 and have had a proven impact but also in your views on burgeoning methods which, while they are not quite ready for prime time, are worth watching.

To nominate a candidate method, you must reference a paper or a webpage describing the method, so that other viewers can see exactly what you mean and express their opinions. It is perfectly fine to put forward your own development, as long as you are upfront about your personal interest.

With one click, you can vote for or against a candidate method and, should you wish to, you also have the ability to post comments. All you need is a free nature.com account. (You do not need a subscription to the journal.) Go to http://www.nature.com/nmeth/votemoy2008 and vote away!

We look forward to hearing about your Method of the Year!

\section{Target practice}

A constant influx of new methods keeps research on microRNA biology fast-paced and can provide divergent vantage points.

The field of microRNA (miRNA) biology is now a little over a decade old, and shows very little sign of flagging vigor. In particular, the adoption of new methodology by scientists in the field continues apace.

miRNAs post-transcriptionally regulate gene expression, typically by destabilizing mRNA or repressing its translation. The role of these regulators in development and disease is now well established, but there is still much to be learned. Central to the effort to understand miRNA function is the identification of the messages that they target.

The first miRNAs were uncovered by genetic analyses in Caenorhabditis elegans, and their targets were accordingly identified as suppressors of loss-of-function miRNA mutants. Then, with the early observation that miRNAs pair to complementary sequences in the $3^{\prime}$ untranslated regions of the messages they regulate, computational methods for target prediction soon began to fly fast and furious. Variably based on sequence features, evolutionary conservation, RNA structure and thermodynamic considerations, the prediction algorithms are still evolving and gaining in power. However, in silico methods are still not sufficient to unambiguously identify functional targets.

In the past few years an assortment of new technologies has been applied to the question of miRNA target prediction. Gene expression microarrays, for instance, have been used to examine miRNA effects on cellular mRNA levels. This has allowed the identification of sequence features enriched within affected messages - information that has been incorporated into the newest wave of target-prediction algorithms.

Microarrays, however, are only informative about effects at the mRNA level. Taking the analysis to the protein level with the application of quantitative proteomics, the laboratories of David Bartel and Nikolaus Rajewsky, each in collaboration with mass spectrometry experts, have now added to the list of techniques used in this field (see Research Highlight, p. 753). The two groups have independently determined what 
happens to thousands of proteins in mouse and human cells when an miRNA is removed, overexpressed or knocked down. They report that the changes in protein level and protein synthesis are overall quite small, but significant. Perhaps more surprisingly, their comparisons of proteomic and microarray data indicate that only a few proteins are substantially and exclusively regulated at the level of translation, although there are some interesting exceptions and more may come to light with additional studies. It is worth noting that, as in the case of microarray experiments, the proteomic data are informative of trends and average properties. Establishing the functional importance of a relationship between an miRNA and a particular message or protein always needs verification.

Coming at the problem from a completely different angle, Pamela Green and her group, in collaboration with Blake Meyers and his team, have applied yet another approach. In a recently published study (German, M. et al., Nat. Biotechnol.26, 941-946; 2008), they prepared libraries containing the products of miRNA-directed mRNA cleavage in Arabidopsis thaliana, sequenced them with next-generation technology and mapped the sequence reads back to the genome. This allowed them to identify not only the genes that these cleavage products corresponded to, but also, based on the high sequence complementarity between plant miRNAs and their targets, the putative miRNA that effected the cleavage. The approach will be worth exploring in animals as well, although it may be complicated by differences in mechanism and miRNA-target complementarity rules between plants and animals.

Even direct biochemical methods, not to be outdone, are making their appearance in this hydra-headed field. The past few years have seen a handful of studies in which immunoprecipitation has been used to directly pull down both miRNAs and mRNAs that are physically associated with proteins of the RNA-induced silencing complex (RISC). The largest such dataset so far, about 3,400 mRNAs strong, was defined last year in the worm (Zhang, L. et al., Mol. Cell 28, 598-613; 2007). On page 813 of this issue, Victor Ambros and his group report a new prediction algorithm for miRNA targets based on the sequence features enriched in these immunoprecipitated messages. This algorithm is the first that is based on a dataset of messages physically associated with miRNA-containing ribonucleoproteins. It predicts all the experimentally verified miRNA-mRNA target interactions in the worm, with a lower false positive rate compared to that of current algorithms.

Different methodologies provide windows onto different aspects of miRNA biology. Expression microarrays, for example, will reveal miRNA-driven destabilization of particular messages. In contrast, if physical complexes are examined, as in immunoprecipitation experiments, stable miRNA-mRNA associations will be preferentially detected. In turn, genetic studies, by and large, will uncover miRNA-target relationships that when disrupted cause a major, observable phenotype.

But the more recent 'omic' view, in revealing that some miRNAs subtly but significantly affect the levels of many cellular messages and proteins, brings a different perspective compared to that of traditional genetics, where an interaction must typically contribute to an easily measurable phenotype to be studied. The functional consequences of subtle miRNA-mediated 'tuning' are not clear and are not necessarily easy to define experimentally. But some argue that this tuning is a genuine regulatory mechanism likely to confer an evolutionary advantage - a view supported by the fact that complementary sequences in miRNAs and their putative targets are evolutionarily conserved more frequently than would be expected by chance. Thus the different views out of so many different methodological windows raise new questions about the underlying biology.

Certainly the continued use of multiple methods to study miRNAs will keep the field exciting for some time to come. There are many aspects of miRNA biology that are still little understood, and their study will need new or at least adapted methodologies. Cell typeand tissue-specific expression patterns, sub-cellular distributions, and the relative abundances of miRNAs and their putative targets are all parameters that affect which miRNA and target actually interact in vivo. Direct and indirect effects will need to be teased out. No doubt, there are surprises still in store. 